\title{
Lytic characterization and application of listerial endolysins PlyP40 and PlyPSA in queso fresco
}

Maxwell J. Holle ${ }^{\circ}$ and Michael J. Miller* ${ }^{*}$

\section{Graphical Abstract}

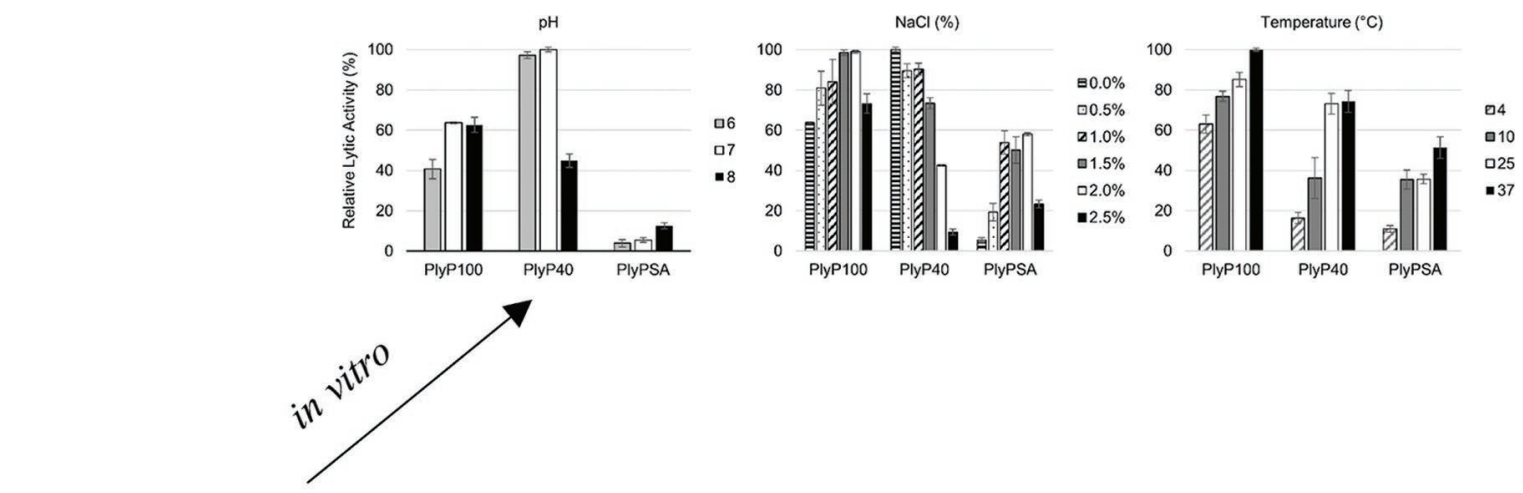

\section{Summary}

Endolysins, cell wall hydrolases derived from bacteriophages, are promising candidates for controlling bacterial pathogens in food systems; however, only a few endolysins have had their lytic capabilities characterized. In this study, changes in lytic activity against Listeria for endolysins PlyP40 and PlyPSA were monitored under varying environmental conditions in vitro and compared with the previously studied endolysin PlyP100. Additionally, the individual endolysins were added to queso fresco (QF) contaminated with Listeria monocytogenes. PlyP40 was able to lower the inoculum cell count over the 28-d shelf life of QF, and PlyPSA was able to lower the cell count until d 14. However, neither endolysin was better than PlyP100 at controlling Listeria monocytogenes in QF.

\section{Highlights}

- PlyP40 had lytic efficacy against a broad range of Listeria

- PlyP40 and PlyPSA were able to maintain lytic activity at refrigeration temperature

- Lytic activity of PlyP40 decreased as pH increased, whereas that of PlyPSA increased

- PlyP40 and PlyPSA maintained lytic activity within the queso fresco (QF) salt range

- PlyP40 and PlyPSA were able to decrease counts of Listeria monocytogenes in QF

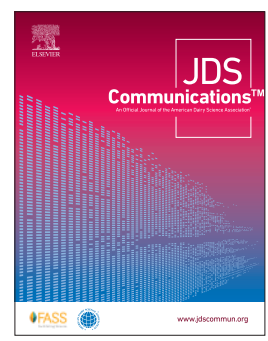

Department of Food Science and Human Nutrition, University of Illinois at Urbana-Champaign, Urbana 61801 . *Corresponding author: mille216@illinois .edu. $\odot$ 2021, The Authors. Published by Elsevier Inc. and Fass Inc. on behalf of the American Dairy Science Association ${ }^{\circledR}$. This is an open access article under the CC BY license (http://creativecommons.org/licenses/by/4.0/). Received July 27, 2020. Accepted December 07, 2020. 


\title{
Lytic characterization and application of listerial endolysins PlyP40 and PlyPSA in queso fresco
}

\author{
Maxwell J. Holle @ and Michael J. Miller* (1)
}

\begin{abstract}
Queso fresco (QF) is a fresh Hispanic-style cheese that is commonly associated with the human foodborne pathogen Listeria monocytogenes and outbreaks of listeriosis in the United States. Endolysins, cell wall hydrolases derived from bacteriophages, are promising candidates for controlling bacterial pathogens in food systems. In this study, we characterized the lytic capabilities of 2 endolysins, PlyP40 and PlyPSA, under varying conditions ( $\mathrm{pH}$, temperature, salt concentration) and compared their activities with those of the previously described endolysin PlyP100. We showed that PlyP40 was effective, showing at least a 33\% reduction in cellular debris, against a broader range of Listeria than PlyPSA, which showed little lytic activity toward Listeria strains not from serovar 4. Both endolysins were also capable of maintaining lytic activity to varying extents at refrigeration temperature. The effect of salt concentration and $\mathrm{pH}$ differed between PlyP40 and PlyPSA. Furthermore, we added the endolysins to QF and monitored their ability to control L. monocytogenes contamination over $28 \mathrm{~d}$ of cold storage. Both PlyP40 and PlyPSA were capable of lowering QF inoculum cell counts compared with the control; however, both were less effective than the previously characterized PlyP100. Further characterization of endolysins will continue to open opportunities to optimization and implementation in a variety of food matrices for controlling pathogen contamination.
\end{abstract}

$L_{\mathrm{p}}^{i}$ isteria monocytogenes is a ubiquitous gram-positive and - psychrotrophic pathogen. Human consumption of food contaminated by L. monocytogenes can lead to listeriosis, especially in at-risk populations such as infants, pregnant women, the elderly, and the immunocompromised. Although L. monocytogenes is a zero-tolerance organism in ready-to-eat foods, dairy products are highly associated with L. monocytogenes contamination in the United States (Batz et al., 2011). Despite most cheeses not being associated with pathogen contamination, Hispanic-style fresh cheeses are frequently contaminated with L. monocytogenes (Ibarra-Sánchez et al., 2017). Almost 20\% of US-reported listeriosis outbreaks between 1998 and 2014 implicated Hispanic-style cheeses, which accounted for $54 \%$ of the total listeriosis cases during this timeframe (Jackson et al., 2018). Queso fresco (QF), the most widely produced fresh Hispanic-style cheese in the United States (Ibarra-Sánchez et al., 2018), is frequently implicated in listeriosis outbreaks (CDC, 2017, Jackson et al., 2018), and has additionally been shown to support $L$. monocytogenes growth, even at $4^{\circ} \mathrm{C}$ (Genigeorgis et al., 1991; Van Tassell et al., 2015; Holle et al., 2018).

Bacteriophage utilize a class of enzymes called endolysins to hydrolyze the host bacterium's peptidoglycan and complete their lytic cycle. Recently, these enzymes have been evaluated for use in several public health situations including food safety (Zhang et al., 2012), diagnostics (Schmelcher et al., 2010), disinfectants (Desbois et al., 2010), and as therapeutic agents (Loeffler et al., 2003; Cheng et al., 2005). Bacteriophages that infect gram-positive bacteria utilize modular endolysins composed of 2 clearly separated functional domains, the enzymatically active domain (EAD) and cell wall binding domain (CBD). The EAD is responsible for the enzymatic mechanism, and there are 5 primary classifications for EADs based upon the particular bond being hydrolyzed (Nelson et al., 2012). The CBD is responsible for binding to the bacterial cell wall and bringing the EAD to the vicinity of its substrate. Currently, Listeria bacteriophage endolysin CBDs have been categorized into 2 primary categories composed of 5 subclasses differing in binding properties such as serovars recognition, distribution on target bacterium, and binding affinity (Schmelcher et al., 2010); however, many remain to be categorized and assessed. In addition, in vitro evaluation of these endolysins has been shown to not fully predict efficacy upon practical application (Ibarra-Sánchez et al., 2018).

However, only a few studies have evaluated an endolysin's lytic efficacy as well as its implementation within a food system for addressing pathogen contamination. Previously, the endolysin PlyP100 was evaluated in a laboratory-scale fresh cheese model (Van Tassell et al., 2017). PlyP40 and PlyPSA are 2 of the only Listeria phage endolysins that have crystal structures (Korndörfer et al., 2006; Romero et al., 2018); however, their lytic activity has not been characterized nor has their implementation in a food matrix been evaluated. The objective of this study was to evaluate and compare the lytic activity of PlyP40 and PlyPSA under varying QF-relevant conditions in vitro and to implement these endolysins into a miniaturized laboratory-scale QF model and compare their efficacy with that of PlyP100.

Individual plasmids containing the gene sequences for PlyP100, PlyP40, and PlyPSA with an accompanying N-terminal His-tag were synthesized by Twist Biosciences (San Francisco, CA). The plasmids were then transformed into competent Escherichia coli BL21 (DE3). Respective cultures were grown aerobically with shaking $(250 \mathrm{rpm})$ at $37^{\circ} \mathrm{C}$ to an optical density (OD) at 595 $\mathrm{nm}\left(\mathrm{OD}_{595}\right)$ of approximately 0.5 before induction with $400 \mu \mathrm{M}$ isopropyl- $\beta$-D-thiogalactoside. Cultures were then incubated for 24 $\mathrm{h}$ at $18^{\circ} \mathrm{C}$, pelleted via centrifugation $4,000 \times g$ for $15 \mathrm{~min}$ at $4^{\circ} \mathrm{C}$, and washed in PBS $\left(\mathrm{KCl}, 200 \mathrm{mg} / \mathrm{L} ; \mathrm{KH}_{2} \mathrm{PO}_{4}, 200 \mathrm{mg} / \mathrm{L} ; \mathrm{NaCl}, 8\right.$ $\mathrm{g} / \mathrm{L} ; \mathrm{Na}_{2} \mathrm{HPO}_{4} 1.15 \mathrm{~g} / \mathrm{L}, \mathrm{pH}$ 7.2). Endolysins were purified from the 
cell pellets using the QIAexpress Ni-NTA Fast Start Kit (Qiagen, Valencia, CA) according to the manufacturer's instructions. Purified endolysins were dialyzed against PBS via Amicon Ultra-15 10 K Centrifugal Filter Unit (10 kDa molecular weight cutoff; Merck Millipore, Burlington, MA) before dilution with an equal volume of glycerol. Before storage $\left(-20^{\circ} \mathrm{C}\right)$, the endolysins were sterilized via filtration. Purity and concentration were determined via $15 \%$ (wt/vol) SDS-PAGE and Quick Start Bradford Protein Assay (BioRad Laboratories, Hercules, CA), respectively.

To determine the lytic spectrum of PlyP40 and PlyPSA, each endolysin was evaluated against the cellular debris of a broad collection of Listeria strains. The previously described PlyP100 was also analyzed. Included within the collection were several strains isolated from food outbreaks, including the cheese-relevant strain NRRL B-33104 derived from the Jalisco QF outbreak. Before each lytic assay, listerial strains (Table 1) were recovered from frozen glycerol stocks $\left(-80^{\circ} \mathrm{C}\right)$ and cultured aerobically in brain-heart infusion broth (Becton, Dickinson and Co., Franklin Lakes, NJ) at $37^{\circ} \mathrm{C}$. Cultures were heat-killed, centrifuged, and suspended in PBS. The cellular debris $\left(\mathrm{OD}_{600}=1.0\right)$ was combined $1: 1$ in a microtiter plate with a final concentration of $2.5 \mu \mathrm{g}$ of each endolysin. Cellular debris combined 1:1 with PBS served as a control in each experiment. The change in $\mathrm{OD}_{600}$ was measured over a 30 -min period using a microplate reader (FilterMax F5 Multi-Mode Microplate Reader, Molecular Devices, San Jose, CA), held at $37^{\circ} \mathrm{C}$.

All L. monocytogenes strains, spanning multiple serovars, were lysed by PlyP100, PlyP40, and PlyPSA. Whereas PlyP100 showed the greatest activity across all of the strains, PlyPSA showed the least (Table 1). For 10 of the 21 strains (48\%), PlyPSA was able to reduce the turbidity by more than $15 \%$. The greatest reductions for PlyPSA were observed against $L$. monocytogenes serovar group 4. Comparatively, however, PlyP100 was able to show at least a
$29.26 \% \pm 1.26 \%$ reduction against all strains, with the highest observed activity against the laboratory strain $10403 \mathrm{~S}$ of L. monocytogenes (serovar 1/2a). Although PlyP40 showed a comparable range of reductions to that of PlyP100, its highest activity only resulted in a $44.34 \% \pm 1.00 \%$ reduction over 30 min. All 3 endolysins also showed activity against Listeria innocua, Listeria ivanovii, and Listeria seeligeri.

Although activity of PlyPSA was lower than that of PlyP40 and PlyP100, it was still active against all tested strains. However, it showed lesser activity against Listeria $1 / 2$ and 3 serovars. This suggests that PlyPSA may not be a strong candidate for food safety use, because $L$. monocytogenes serovars $1 / 2 \mathrm{a}$ and $1 / 2 \mathrm{~b}$ are the 2 serovars most commonly implicated in human foodborne illness (Kathariou, 2002). The lytic spectrum for both PlyPSA and PlyP40 has not been extensively evaluated before this study; however, the binding spectrums for the CBD of both PlyPSA and PlyP40 alone corroborate their lytic spectrums (Schmelcher et al., 2010). Previously, Schmelcher et al. (2010) showed that PlyP40 binds strongly to all serovars of Listeria, whereas PlyPSA showed little to no binding for serovars $1 / 2 \mathrm{a}, 1 / 2 \mathrm{~b}, 3 \mathrm{a}, 4 \mathrm{~b}, 3 \mathrm{c}$, and 7 .

Endolysin activity is modulated by key, food-relevant environmental factors such as $\mathrm{pH}, \mathrm{NaCl}$ concentration, and temperature. To determine how the lytic activity of each endolysin would be affected by these environmental factors, $2.5 \mu \mathrm{g}$ of each endolysin was tested against L. innocua (ATCC 33090) cellular debris under varying QF-relevant $\mathrm{pH}$ values (6-8), $\mathrm{NaCl}$ concentrations $(0.0-2.5 \%)$, and temperatures $\left(4,25\right.$, and $37^{\circ} \mathrm{C}$; Ibarra-Sánchez et al., 2017; Holle et al., 2018), as previously described (Van Tassell et al., 2017). Briefly, for $\mathrm{pH}$ and salt lytic assays, $40 \mathrm{mM}$ boric acid/phosphoric acid buffer (BP) was used in place of PBS buffer and adjusted to the corresponding $\mathrm{pH}$ levels (6-8). The $\mathrm{NaCl}$ experiments also used BP buffer $(\mathrm{pH} 7)$ with different amounts of

Table 1. Activity (as shown by reduction in turbidity) of endolysins PlyP100, PlyP40, and PlyPSA against different Listeria species $^{1}$

\begin{tabular}{|c|c|c|c|c|c|}
\hline \multirow[b]{2}{*}{ Species } & \multirow[b]{2}{*}{ Strain ID } & \multirow[b]{2}{*}{ Serovar } & \multicolumn{3}{|c|}{ Reduction in turbidity (\%) } \\
\hline & & & PlyP100 & PlyP40 & PlyPSA \\
\hline \multirow[t]{18}{*}{ L. monocytogenes } & $10403 S$ & $1 / 2 a$ & $52.49 \pm 0.30$ & $36.12 \pm 1.00$ & $2.92 \pm 0.93$ \\
\hline & SLCC-5764 & $1 / 2 a$ & $33.87 \pm 0.51$ & $34.38 \pm 0.73$ & $11.86 \pm 1.71$ \\
\hline & NRRL B-33419² & $1 / 2 a$ & $45.9 \pm 0.60$ & $38.68 \pm 0.89$ & $6.58 \pm 1.40$ \\
\hline & NRRL B-33395 & $1 / 2 \mathrm{a}$ & $44.95 \pm 0.37$ & $40.72 \pm 1.69$ & $8.19 \pm 1.89$ \\
\hline & NRRL B-33391 & $1 / 2 b$ & $44.61 \pm 0.43$ & $29.07 \pm 0.81$ & $5.37 \pm 0.62$ \\
\hline & NRRL B-33424² & $1 / 2 b$ & $47.32 \pm 0.33$ & $44.34 \pm 1.00$ & $4.96 \pm 1.28$ \\
\hline & ATCC 7644 & $1 / 2 c$ & $39.68 \pm 0.12$ & $32.62 \pm 1.38$ & $11.37 \pm 0.62$ \\
\hline & ATCC 19112 & $1 / 2 c$ & $40.9 \pm 0.48$ & $37.98 \pm 0.72$ & $5.71 \pm 0.77$ \\
\hline & NRRL B-33393 & $3 b$ & $44.42 \pm 0.49$ & $35.99 \pm 2.13$ & $7.89 \pm 1.51$ \\
\hline & NRRL B-33226 & $3 c$ & $42.29 \pm 0.27$ & $37.79 \pm 0.99$ & $4.60 \pm 1.20$ \\
\hline & NRRL B-33403 & $4 a$ & $48.88 \pm 0.20$ & $36.33 \pm 1.69$ & $35.98 \pm 5.17$ \\
\hline & ATCC 13932 & $4 b$ & $37.93 \pm 0.68$ & $28.95 \pm 0.57$ & $26.47 \pm 2.00$ \\
\hline & NRRL B-33420² & $4 b$ & $41.44 \pm 0.60$ & $38.31 \pm 1.00$ & $20.78 \pm 2.99$ \\
\hline & NRRL B-33513² & $4 b$ & $38.41 \pm 0.29$ & $17.67 \pm 4.66$ & $27.36 \pm 0.79$ \\
\hline & NRRL B-33104² & $4 b$ & $48.58 \pm 0.26$ & $35.57 \pm 0.95$ & $23.23 \pm 3.48$ \\
\hline & NRRL B-33231 & $4 c$ & $41.10 \pm 1.51$ & $38.52 \pm 0.99$ & $31.17 \pm 4.23$ \\
\hline & NRRL B-33116 & $4 d$ & $39.32 \pm 0.87$ & $35.11 \pm 1.27$ & $15.95 \pm 0.30$ \\
\hline & NRRL B-33120 & $4 \mathrm{e}$ & $37.76 \pm 0.25$ & $34.26 \pm 1.36$ & $25.82 \pm 4.69$ \\
\hline L. innocua & ATCC 33090 & $6 a$ & $44.85 \pm 0.31$ & $33.36 \pm 2.45$ & $23.01 \pm 2.44$ \\
\hline L. ivanovii ssp. ivanovii & NRRL B-33017 & 5 & $29.26 \pm 1.26$ & $35.03 \pm 1.52$ & $34.20 \pm 0.04$ \\
\hline L. seeligeri & NRRL B-33019 & $1 / 2 b$ & $49.16 \pm 0.49$ & $38.31 \pm 2.18$ & $14.67 \pm 1.90$ \\
\hline
\end{tabular}

${ }^{1}$ Values are means \pm SEM for triplicate independent experiments.

${ }^{2}$ Isolated strains associated with food outbreaks. 
$\mathrm{pH}$

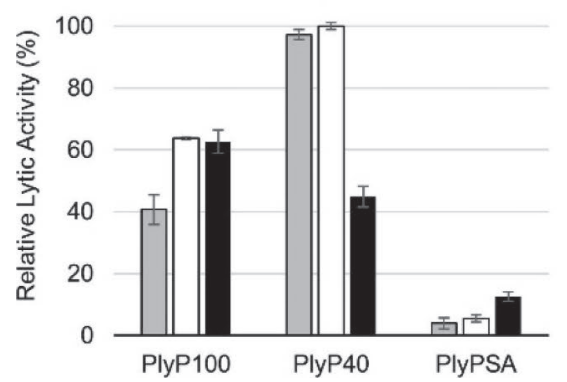

$\mathrm{NaCl}(\%)$

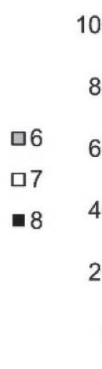

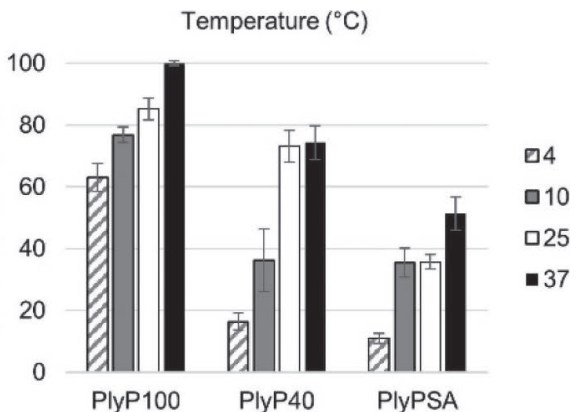

Figure 1. Optimal queso fresco-relevant environmental conditions for the lytic activity of endolysins PlyP100, PlyP40, and PlyPSA. The optimal (A) pH, (B) NaCl, and (C) temperature conditions for the lysis of heat-killed Listeria innocua ATCC 33090 was characterized via turbidity reduction assay over 30 min using 2.5 $\mu \mathrm{g} / \mathrm{mL}$ concentrations of each endolysin. The highest reduction value across all 3 endolysins was set to $100 \%$ for comparison. Bars indicate the mean \pm SEM for triplicate independent experiments.

$\mathrm{NaCl}$ dissolved therein $(0-2.5 \% \mathrm{wt} / \mathrm{vol})$. To evaluate the effect of temperature on the lytic activity of the endolysins, all assay materials were incubated at different temperatures $\left(4,25\right.$, or $\left.37^{\circ} \mathrm{C}\right)$.

The effect of $\mathrm{pH}$ and salt concentration on lytic activity of each endolysin was evaluated individually within QF-appropriate ranges ( $\mathrm{pH} 6-8, \mathrm{NaCl} 1.0-2.5 \%$; Figure 1). The lytic activity of each endolysin was evaluated in $\mathrm{BP}$ buffer over a $\mathrm{pH}$ range from 6.0 to 8.0. Both PlyP100 and PlyP40 showed the greatest activity around $\mathrm{pH}$ 7. As acidity increased, however, PlyP100 activity decreased, whereas that of PlyP40 did not. At the other end of the spectrum, activity of PlyPSA was highest at $\mathrm{pH} 8$ and showed an approximately $50 \%$ decrease at $\mathrm{pH} 7$. As the acidity increased to $\mathrm{pH} 6$, activity of PlyPSA continued to decrease. The effect of $\mathrm{NaCl}$ on the lytic activity of endolysins was also evaluated within the commonly observed salt concentrations for QF (Holle et al., 2018). All endolysins showed activity within the common QF salt range of 1 to $2 \%$. PlyP 100 maintained its highest activity between 1.5 and $2.0 \% \mathrm{NaCl}(\mathrm{wt} \%$ ), which is the typical range for $\mathrm{QF}$ (Holle et al., 2018). As the salt concentration increased, lytic activity of PlyP40 began to decrease, with approximately one-tenth of its ac-

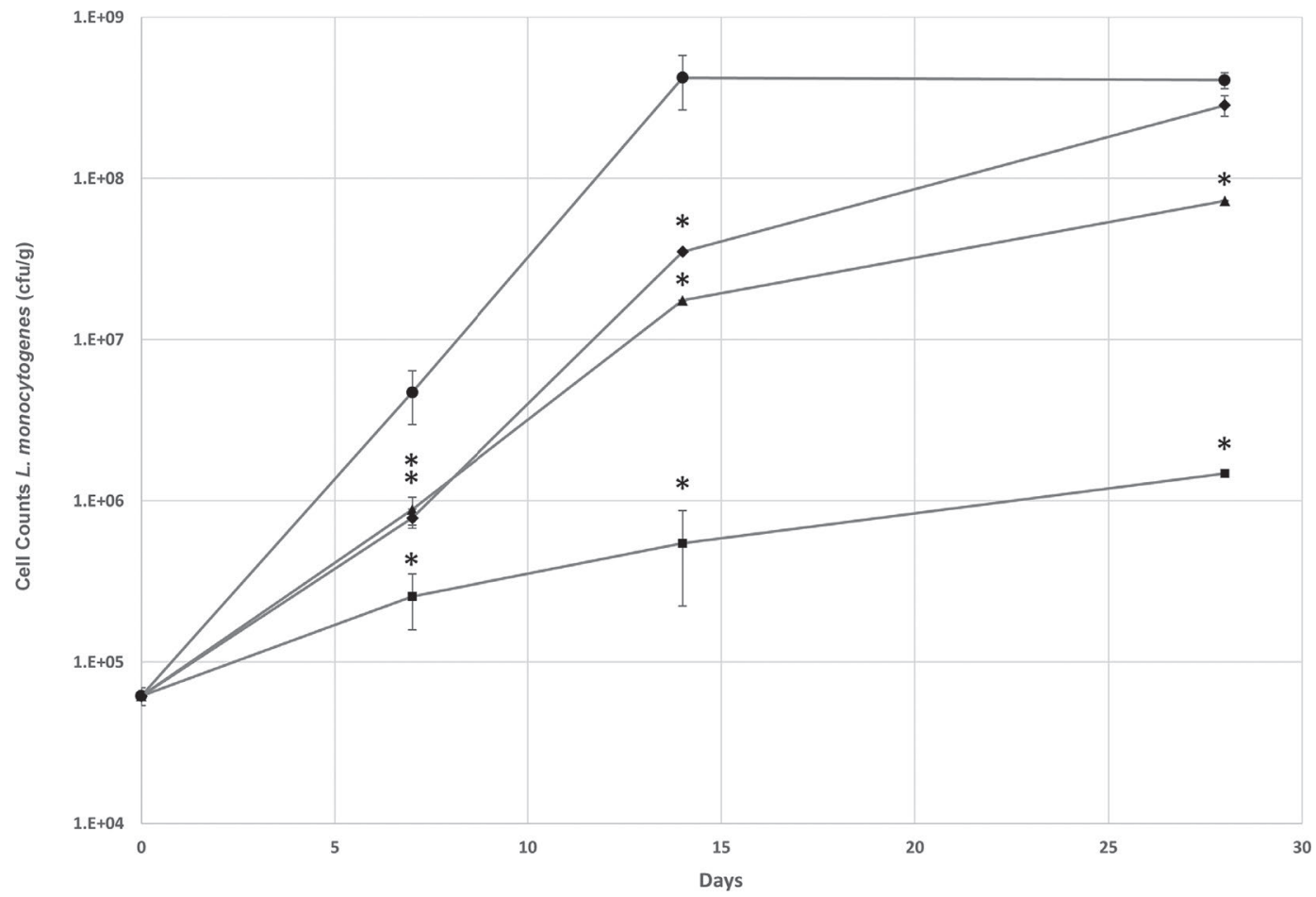

Figure 2. Antimicrobial activity of endolysins PlyP100 ( $\mathbf{\square})$, PlyP40 ( $\mathbf{\Delta})$, and PlyPSA ( $)$, compared with a PBS control $(\bullet)$ against Listeria monocytogenes NRRL B-33104 in a miniaturized laboratory-scale queso fresco over $28 \mathrm{~d}$ of storage at $4^{\circ} \mathrm{C}$. Each endolysin was added at $250 \mu \mathrm{g} / \mathrm{g}$ of cheese. Values are means \pm SEM for triplicate independent experiments. One-way ANOVA was performed, and the antimicrobial effect compared with the control was evaluated using Tukey's test to determine statistical significance $(a<0.05)$ of mean differences. Asterisks indicate significant difference $(P<0.05)$ compared with the control. 
tivity remaining at $2.5 \% \mathrm{NaCl}$. In contrast, the activity of PlyPSA decreased when the salt concentration was $<1 \%$ or $>2 \%$.

We evaluated the long-term stability of the endolysins to evaluate their shelf life stability. Each endolysin $(250 \mu \mathrm{g})$ was held at 4 and $25^{\circ} \mathrm{C}$ in PBS for $28 \mathrm{~d}$ while evaluating its activity every $7 \mathrm{~d}$, as previously described (Loeffler et al., 2003). At each sampling point, the lytic activity against L. innocua (ATCC 33090) debris was measured as described above. We observed no decrease in lytic activity among any of the endolysins (data not shown).

Food matrices are complex beyond the simple parameters evaluated in vitro; thus, each endolysin was incorporated into a fresh cheese model inoculated with L. monocytogenes (NRRL B-33104). The cheeses were prepared as previously described (Van Tassell et al., 2017), and PlyP40, PlyPSA, and PlyP100 were each added to the drained curd of their respective cheeses at a final concentration of $250 \mu \mathrm{g} / \mathrm{g}$ of cheese. Data were analyzed by one-way ANOVA, and the antimicrobial effect compared with the control was evaluated using Tukey's test to determine statistical significance $(\alpha<$ 0.05) of mean differences using the data analysis add-on in Microsoft Excel (Redmond, WA). Asterisks indicate significant difference compared with the control.

The L. monocytogenes counts from the untreated miniaturized laboratory queso fresco (MLQF)showed growth of approximately $4 \mathrm{log} \mathrm{cfu} / \mathrm{g}$ after $28 \mathrm{~d}$ with a final level of $\sim 8.6 \mathrm{log} \mathrm{cfu} / \mathrm{g}$ (Figure 2). PlyPSA hindered the growth of $L$. monocytogenes in the MLQF; however, after $28 \mathrm{~d}$, Listeria counts had increased to $8.5 \log \mathrm{cfu} / \mathrm{g}$, which was not significantly $(\alpha<0.05)$ different from the control. PlyP40 showed a similar effect to PlyPSA, but was more effective, reducing the viable cell counts to only $7.9 \log \mathrm{cfu} / \mathrm{g}$. PlyP100 was the most effective, at the evaluated concentration, at limiting cell counts of the inoculum.

In summary, we evaluated the lytic spectrums for PlyP40 and PlyPSA and both were capable of lysing the lytic debris from all of the tested Listeria strains. The evaluated endolysins all maintained optimal activity at $37^{\circ} \mathrm{C}$; however, the degree of activity lost at colder temperatures varied among the enzymes. Similar to that of PlyP100, the activity of PlyPSA increased as $\mathrm{pH}$ increased, whereas PlyP40 exhibited the opposite behavior. PlyP40 was the most effective enzyme at lower salt concentrations, suggesting that there may be situations in which it is the optimal Listeria phage endolysin compared with PlyP100 and PlyPSA. PlyP100 was still the most effective endolysin at controlling L. monocytogenes in $\mathrm{QF}$. Future work could focus on further evaluating situations in which PlyP40 may be an optimal endolysin. Additionally, further insight into the functional capacity of PlyP100 EAD and CBD should elucidate the observed effectiveness. Genetic manipulations (e.g., domain swapping) may further increase our understanding of these endolysins and increase their ability to be applied for food safety applications.

\section{References}

Batz, M. B., S. A. Hoffmann, and J. G. Morris. 2011. Ranking the risks: The 10 pathogen-food combinations with the greatest burden on public health. Emerging Pathogens Institute, University of Florida, Gainesville.

CDC. 2017. List of Selected Multistate Foodborne Outbreak Investigations. Vol. 2017. Accessed Feb. 15, 2020. https://www.cdc.gov/foodsafety/ outbreaks/multistate-outbreaks/outbreaks-list.html.

Cheng, Q., D. Nelson, S. Zhu, and V. A. Fischetti. 2005. Removal of group B streptococci colonizing the vagina and oropharynx of mice with a bacte- riophage lytic enzyme. Antimicrob. Agents Chemother. 49:111-117. https:/ /doi.org/10.1128/AAC.49.1.111-117.2005.

Desbois, A. P., S. Lang, C. G. Gemmell, and P. J. Coote. 2010. Surface disinfection properties of the combination of an antimicrobial peptide, ranalexin, with an endopeptidase, lysostaphin, against methicillin-resistant Staphylococcus aureus (MRSA). J. Appl. Microbiol. 108:723-730. https://doi.org/ 10.1111/j.1365-2672.2009.04472.x.

Genigeorgis, C., M. Carniciu, D. Dutulescu, and T. B. Farver. 1991. Growth and survival of Listeria monocytogenes in market cheeses stored at 4 to 30 C. J. Food Prot. 54:662-668. https://doi.org/10.4315/0362-028X-54.9.662.

Holle, M. J., L. A. Ibarra-Sánchez, X. Liu, M. J. Stasiewicz, and M. J. Miller. 2018. Microbial analysis of commercially available US Queso Fresco. J. Dairy Sci. 101:7736-7745. https://doi.org/10.3168/jds.2017-14037.

Ibarra-Sánchez, L., M. Van Tassell, and M. Miller. 2017. Invited review: Hispanic-style cheeses and their association with Listeria monocytogenes. J. Dairy Sci. 100:2421-2432. https://doi.org/10.3168/jds.2016-12116.

Ibarra-Sánchez, L. A., M. L. Van Tassell, and M. J. Miller. 2018. Antimicrobial behavior of phage endolysin PlyP100 and its synergy with nisin to control Listeria monocytogenes in Queso Fresco. Food Microbiol. 72:128-134. https://doi.org/10.1016/j.fm.2017.11.013.

Jackson, K. A., L. H. Gould, J. C. Hunter, Z. Kucerova, and B. Jackson. 2018. Listeriosis outbreaks associated with soft cheeses, United States, 19982014. Emerg. Infect. Dis. 24:1116-1118. https://doi.org/10.3201/eid2406 .171051 .

Kathariou, S. 2002. Listeria monocytogenes virulence and pathogenicity, a food safety perspective. J. Food Prot. 65:1811-1829. https://doi.org/10.4315/ 0362-028X-65.11.1811.

Korndörfer, I. P., J. Danzer, M. Schmelcher, M. Zimmer, A. Skerra, and M. J. Loessner. 2006. The crystal structure of the bacteriophage PSA endolysin reveals a unique fold responsible for specific recognition of Listeria cell walls. J. Mol. Biol. 364:678-689. https://doi.org/10.1016/j.jmb.2006.08 .069 .

Loeffler, J. M., S. Djurkovic, and V. A. Fischetti. 2003. Phage lytic enzyme Cp11 as a novel antimicrobial for pneumococcal bacteremia. Infect. Immun. 71:6199-6204. https://doi.org/10.1128/IAI.71.11.6199-6204.2003.

Nelson, D. C., M. Schmelcher, L. Rodriguez-Rubio, J. Klumpp, D. G. Pritchard, S. Dong, and D. M. Donovan. 2012. Endolysins as Antimicrobials. Adv. Virus Res. 83:299-365. https://doi.org/10.1016/B978-0-12-394438-2 $.00007-4$.

Romero, P., S. G. Bartual, M. Schmelcher, C. Glück, J. A. Hermoso, and M. J. Loessner. 2018. Structural insights into the binding and catalytic mechanisms of the Listeria monocytogenes bacteriophage glycosyl hydrolase PlyP40. Molecular Microbiol. 108:128-142. https://doi.org/10.1111/mmi .13922 .

Schmelcher, M., T. Shabarova, M. R. Eugster, F. Eichenseher, V. S. Tchang, M. Banz, and M. J. Loessner. 2010. Rapid multiplex detection and differentiation of Listeria cells by use of fluorescent phage endolysin cell wal binding domains. Appl. Environ. Microbiol. 76:5745-5756. https://doi.org/ 10.1128/AEM.00801-10.

Van Tassell, M. L., L. A. Ibarra-Sánchez, G. P. Hoepker, and M. J. Miller. 2017. Hot topic: Antilisterial activity by endolysin PlyP100 in fresh cheese. J. Dairy Sci. 100:2482-2487. https://doi.org/10.3168/jds.2016-11990.

Van Tassell, M. L., L. A. Ibarra-Sánchez, S. R. Takhar, S. L. Amaya-Llano, and M. J. Miller. 2015. Use of a miniature laboratory fresh cheese model for investigating antimicrobial activities. J. Dairy Sci. 98:8515-8524. https:// doi.org/10.3168/jds.2015-9967.

Zhang, H., H. Bao, C. Billington, J. A. Hudson, and R. Wang. 2012. Isolation and lytic activity of the Listeria bacteriophage endolysin LysZ5 against Listeria monocytogenes in soya milk. Food Microbiol. 31:133-136. https:/ /doi.org/10.1016/j.fm.2012.01.005.

\section{Notes}

Maxwell J. Holle @ https://orcid.org/0000-0003-0328-2982 Michael J. Miller @ https://orcid.org/0000-0001-8579-1080

This study was supported by the USDA Cooperative State Research, Education and Extension Service (Washington, DC; Hatch project \#ILLU-698-339 to MJM). The University of Illinois College of ACES provided support for the first author.

The authors have not stated any conflicts of interest. 